\title{
FRECUENCIA Y PATOTIPOS DE Escherichia coli DIARROGÉNICA EN NIÑOS PERUANOS CON Y SIN DIARREA
}

\author{
Theresa J. Ochoa ${ }^{1,2, a}$, Erik H. Mercado ${ }^{1, b}$, David Durand ${ }^{1, b}$, Fulton P. Rivera ${ }^{1, c}$, Susan Mosquito ${ }^{1, d}$, \\ Carmen Contreras ${ }^{1, \mathrm{e}}$, Maribel Riveros ${ }^{1, \mathrm{~b}}$, Angela Lluque $^{1, \mathrm{~b}}$, Francesca Barletta ${ }^{1, \mathrm{e}}$, \\ Ana Prada ${ }^{1, d}$, Joaquim Ruiz ${ }^{3, d}$
}

\begin{abstract}
RESUMEN
Introducción. Las E. coli diarrogénicas (DEC) son una de las principales causas de diarrea en niños en países en vías de desarrollo. Sin embargo, no son rutinariamente diagnosticadas en los laboratorios clínicos. Objetivos. Determinar la prevalencia de las DEC en niños peruanos y describir la variabilidad genética de estas cepas. Materiales y métodos. Se utilizaron 8003 cepas de E. coli previamente aisladas de ocho estudios previos de diarrea en niños, mayormente en zonas periurbanas de Lima. El diagnóstico de las DEC fue a través de un PCR múltiple a tiempo real para los seis grupos de DEC. Se empleó PCR para la determinación de genes adicionales de virulencia. Resultados. La prevalencia promedio global en muestras de diarrea $(n=4243)$ fue: $E$. coli enteroagregativa (EAEC) $9,9 \%$, enteropatogénica (EPEC) $8,5 \%$, enterotoxigénica (ETEC) $6,9 \%$, difusamente adherente (DAEC) $4,8 \%$, productora de toxina shiga (STEC) $0,8 \%$ y enteroinvasiva (EIEC) $0,6 \%$. La frecuencia relativa de cada patógeno varía según la edad y tipo de estudio. Los principales patotipos en muestras control $(n=3760)$ fueron EPEC $(10,9 \%)$ y EAEC $(10,4 \%)$. Se encontró una gran variabilidad en la frecuencia de genes de virulencia para cada patotipo, así como en los mecanismos moleculares de resistencia, sin diferencias significativas entre muestras de diarrea y control. Conclusiones. Las DEC son causa importante de diarrea en niños peruanos. Estos patógenos son altamente heterogéneos. Se requieren estudios adicionales para determinar la prevalencia en zonas rurales del Perú, así como en casos graves de diarrea.
\end{abstract}

Palabras clave: Escherichia coli, Diarrea, Niño, Perú, Virulencia. (fuente: DeCS BIREME).

\section{FREQUENCY AND PATHOTYPES OF DIARRHEAGENIC Escherichia coli IN PERUVIAN CHILDREN WITH AND WITHOUT DIARRHEA}

\begin{abstract}
Introduction. Diarrheagenic E. coli (DEC) are a major cause of diarrhea in children in developing countries. However, they are not part of routine diagnosis in clinical laboratories. Objectives. To determine the DEC prevalence in Peruvian children and to describe the genetic variability of these strains. Materials and methods. A total of $8003 \mathrm{E}$. coli strains previously isolated from eight different studies of diarrhea in children, mainly from peri-urban areas of Lima, were analyzed. Diagnosis of DEC was done with Multiplex real-time PCR using genes for each of the 6 DEC groups. Conventional PCR was performed for the detection of additional virulence genes. Results. Globally, the mean prevalence in diarrhea samples $(n=4,243)$ was: enteroaggregative E. coli (EAEC) 9.9\%, enteropathogenic E. coli (EPEC) 8.5\%, enterotoxigenic E. coli (ETEC) $6.9 \%$, diffusely adherent $E$. coli (DAEC) $4.8 \%$, Shiga toxin-producing E. coli (STEC) $0.8 \%$ and enteroinvasive E. coli (EIEC) $0.6 \%$. The relative frequency of each pathogen varies according to the age and the type of study. The main pathotypes in control samples $(n=3,760)$ were EPEC $(10.9 \%)$ and EAEC (10.4\%). An important variability in the virulence genes frequency and molecular resistance mechanisms for each pathotype was found, without differences between diarrhea and control groups. Conclusions. DEC are a major cause of diarrhea in Peruvian children. These pathogens are highly heterogeneous. Additional studies are required to determine the prevalence in rural areas of Peru and in severe diarrhea cases.
\end{abstract}

Key words: Escherichia coli, Diarrhea, Child, Peru, Virulence. (source: MeSH NLM).

\section{INTRODUCCIÓN}

La diarrea continúa siendo un importante problema de salud en el mundo, especialmente en los países en vías de desarrollo (1). Entre las bacterias asociadas a diarrea infantil están los diferentes patotipos de las $E$. coli diarrogénicas (DEC). Estas bacterias colonizan el intestino del ser humano, pueden transmitirse directamente de persona a persona, de animal a persona o indirectamente a través del agua o los alimentos contaminados. Según su patogénesis y las características epidemiológicas, este grupo de bacterias se divide en seis patotipos: $E$. coli enteropatógena (EPEC, por sus siglas en inglés Enteropathogenic E.coli),

\footnotetext{
Instituto de Medicina Tropical "Alexander von Humboldt”, Universidad Peruana Cayetano Heredia, Lima, Perú.

2 University of Texas School of Public Health, Houston, Texas, Estados Unidos.

3 Centre de Recerca en Salut Internacional, Hospital Clinic / IDIBAPS, Universitat de Barcelona, España.

a Médico Infectólogo Pediatra; ' Biólogo; ' Tecnólogo Médico; ' Microbiólogo; e Biólogo Molecular.
}

Recibido: 17-01-11 Aprobado: 09-02-11 
productora de toxina shiga (STEC), enterotoxigénica (ETEC), enteroinvasiva (EIEC), enteroagregativa (EAEC) y difusamente adherente (DAEC) (2). El diagnóstico de estos patógenos se puede realizar por serología (determinando los serotipos comúnmente asociados a cada patotipo, por ejemplo serotipos clásicos de EPEC), por cultivo celular (identificando un patrón de adherencia característico, por ejemplo para EPEC y EAEC), por ELISA (identificando la presencia de toxinas, por ejemplo LT y ST para ETEC o toxina shiga para STEC), o por métodos moleculares (identificando genes de virulencia por reacción en cadena de la polimerasa [PCR] para cada uno de los patotipos). De todos estos métodos, el último, se ha convertido en la prueba estándar usada para el diagnóstico. Recientemente nuestro grupo de investigación ha desarrollado y validado un PCR múltiple a tiempo real (mRT-PCR) para la detección de los seis grupos de DEC ${ }^{(3)}$.

Las DEC en conjunto son responsables del 30 a $40 \%$ de los episodios de diarrea aguda en niños en países en vías de desarrollo ${ }^{(4)}$. En una revisión reciente para la Organización Mundial de la Salud, se encontró que EPEC y ETEC fueron consideradas como alta prioridad para el desarrollo de vacunas luego de rotavirus, por sus altas tasas de mortalidad y morbilidad ${ }^{(5)}$. Por lo que es importante tener datos locales y regionales de la distribución de estos patógenos. En el Perú, las DEC han sido descritas en estudios previos tanto en población pediátrica como en adultos, haciendo uso de diversos métodos diagnósticos ${ }^{(6,7,8)}$. Sin embargo, no se han buscado de manera sistemática los seis grupos actualmente reconocidos. El objetivo de este trabajo fue determinar la prevalencia de las $D E C$ en niños peruanos y describir la variabilidad genética de estas cepas.

\section{MATERIALES Y MÉTODOS}

\section{POBLACIÓN Y MUESTRA}

Para la determinación de la prevalencia de las DEC en el Perú, se utilizaron cepas de E. coli previamente aisladas de 3284 pacientes pediátricos de ocho estudios previos (Tabla 1). Se incluyeron estudios comunitarios de diarrea (estudios \# 1, 2, 4, 5 y 8), así como estudios hospitalarios (estudios \# 3, 6 y 7). Todos, excepto uno (estudio \# 1), fueron estudios recientes, del 2004 en adelante. Todos los estudios, excepto uno (estudio \# 7) incluyeron población sana menor de 5 años. El estudio de Medina y colaboradores (estudio \# 7) (11) incluyó pacientes hasta los 18 años y fue exclusivamente en pacientes infectados con el virus de inmunodeficiencia humana $(\mathrm{VIH})$. En los estudios de cohorte no solo se colectaron muestras de diarrea, sino también muestras control en ausencia de diarrea (estudio \# 1, 4, 5, 7 y 8). Estas muestras permiten medir el grado de colonización asintomática por estos patógenos entéricos.

\section{PROCEDIMIENTOS}

Entre los años 2005 y 2010 se analizaron 8003 muestras. Por cada muestra se contó con una a cinco colonias de $E$. coli guardadas en peptonas de estudios anteriores (estudios \#1-3, Tabla 1) así como colonias de E. coli colectadas de placas de Mac Conkey de

Tabla 1. Estudios clínicos y epidemiológicos de diarrea en niños peruanos en los que se diagnosticaron las E. coli diarrogénicas por métodos moleculares.

\begin{tabular}{|c|c|c|c|c|c|c|}
\hline N. ${ }^{\circ}$ & $\begin{array}{l}\text { Investigadores } \\
\text { del estudio }\end{array}$ & Lugar & Año & Edad & $\begin{array}{l}\text { N. }{ }^{\circ} \text { de } \\
\text { niños }\end{array}$ & Descripción del estudio \\
\hline 1 & Lanata CF & Huaraz, Ancash & $\begin{array}{l}1986- \\
1987\end{array}$ & $0-36 m$ & 285 & $\begin{array}{l}\text { Estudio cohorte. Vigilancia activa de } \\
\text { diarrea en la comunidad. }\end{array}$ \\
\hline 2 & Zavaleta N & Villa el Salvador, Lima & 2004 & $6 m-18 m$ & 313 & $\begin{array}{l}\text { Estudio cohorte. Vigilancia activa de } \\
\text { diarrea en la comunidad (ensayo clínico). }\end{array}$ \\
\hline 3 & Zavaleta N & $\begin{array}{l}\text { Instituto Nacional de Salud del } \\
\text { Niño, Lima }\end{array}$ & 2005 & $4 m-36 m$ & 120 & $\begin{array}{l}\text { Niños hospitalizados con diarrea aguda y } \\
\text { deshidratación (ensayo clínico) }{ }^{(9)} \text {. }\end{array}$ \\
\hline 4 & $\begin{array}{l}\text { Ochoa TJ y Lanata } \\
\text { CF }\end{array}$ & $\begin{array}{l}\text { Chrorrillos, Villa el Salvador, Villa } \\
\text { María del Triunfo, San Juan de } \\
\text { Miraflores, Lima }\end{array}$ & $\begin{array}{l}2006- \\
2007\end{array}$ & $2 m-12 m$ & 1034 & $\begin{array}{l}\text { Estudio cohorte. Vigilancia pasiva de } \\
\text { diarrea en la comunidad (10). }\end{array}$ \\
\hline 5 & $\begin{array}{l}\text { Ochoa TJ, Lanata } \\
\text { CF y Huicho L }\end{array}$ & Chorrillos, Lima & 2008 & $13 m-20 m$ & 529 & $\begin{array}{l}\text { Estudio cohorte. Vigilancia pasiva de } \\
\text { diarrea en la comunidad. }\end{array}$ \\
\hline 6 & $\begin{array}{l}\text { Llanos A, Lee J y } \\
\text { Lopez F }\end{array}$ & $\begin{array}{l}\text { Hospital Nacional Cayetano } \\
\text { Heredia, Lima }\end{array}$ & $\begin{array}{l}2007- \\
2008\end{array}$ & $0-5 a$ & 135 & $\begin{array}{l}\text { Niños atendidos en la Emergencia por } \\
\text { diarrea con sangre. }\end{array}$ \\
\hline 7 & $\begin{array}{l}\text { Medina A, Rivera } \\
\text { FP y Romero L. }\end{array}$ & $\begin{array}{l}\text { Instituto Nacional de Salud del } \\
\text { Niño, Hosp. Nacional Cayetano } \\
\text { Heredia y Hosp. Nacional } \\
\text { Hipólito Unanue }\end{array}$ & $\begin{array}{l}2007- \\
2009\end{array}$ & $1 m-18 a$ & 113 & $\begin{array}{l}\text { Niños con VIH atendidos en la } \\
\text { Emergencia o en Consultorio Externo (11). }\end{array}$ \\
\hline 8 & $\begin{array}{l}\text { Ochoa TJ, Chea E } \\
\text { y Cleary }\end{array}$ & Independencia, Lima & $\begin{array}{l}2008- \\
2010\end{array}$ & $12 m-24 m$ & 555 & $\begin{array}{l}\text { Estudio cohorte. Vigilancia activa de } \\
\text { diarrea en la comunidad (ensayo clínico). }\end{array}$ \\
\hline
\end{tabular}




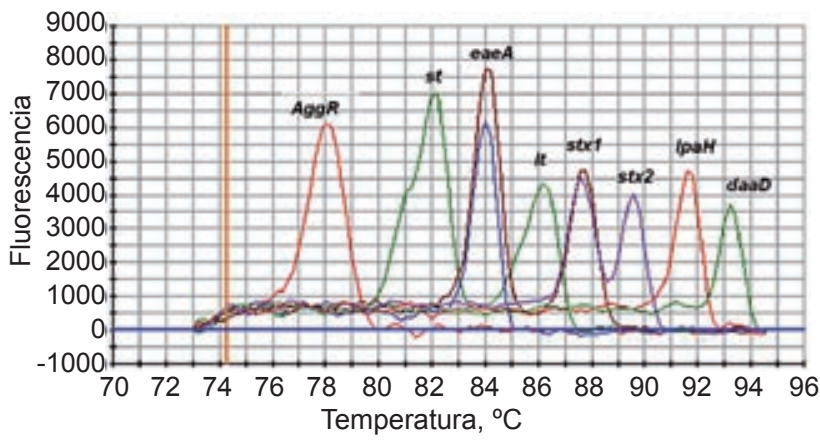

Figura 1. PCR múltiple a tiempo real (mRT-PCR) para la detección simultánea de los genes de virulencia de $E$. coli diarrogénicas: EAEC $(A g g R)$, ETEC (st, It), EPEC (eaeA), STEC (stx1, stx2), EIEC (ipaH) y DAEC (daaD). Los datos corresponden a tubos individuales de reacción con ADN de cada cepa. Se muestran las curvas de denaturación de los respectivos genes amplificados. El eje $Y$ (Fluorescencia) representa la derivada negativa de la fluorescencia sobre la temperatura versus la temperatura en grados centígrados.

coprocultivos en curso de estudios recientes (estudios \#4-8, Tabla 1).

Para la determinación de las DEC se usó el mRT-PCR, el cual fue implementado y validado en el Laboratorio de Enfermedades Entéricas y Nutrición (LEEN) del Instituto de Medicina Tropical de la Universidad Peruana Cayetano Heredia en Lima, desde el año 2005. Este PCR utiliza fluorescencia para determinar la temperatura de denaturación de cada amplicon basado en la disociación del SYBER Green. Los primers o cebadores han sido diseñados para reconocer simultáneamente nueve genes en una sola reacción: aggR (EAEC), st1, st2, It (ETEC), eae (EPEC), eae, stx1, stx2 (STEC), ipaH (EIEC), y daaD $\left(\right.$ DAEC) ${ }^{(3)}$ (Figura 1). Para esta prueba se seleccionan al azar cinco colonias lactosa positivas de la placa de Mac Conkey, para la extracción de DNA y posterior PCR. Para hacer la prueba menos costosa para su uso en países en vías de desarrollo, hemos desarrollado y validado un método usando un pool o conglomerado de cinco colonias por paciente/muestra, en vez del análisis de PCR indivi- dual de cada colonia. El análisis del pool, tiene una sensibilidad de $98 \%$ y una especificidad de $100 \%$, a un quinto del costo del análisis individual de colonias ${ }^{(12)}$. Para la determinación de la susceptibilidad antibiótica a quinolonas, betalactámicos, cloranfenicol y tetraciclinas se utilizó la técnica de disco de Kirby Bauer. Para la determinación de los mecanismos de resistencia a antibióticos se realizaron PCR con primers o cebadores dirigidos a genes específicos, según metodología previamente descrita ${ }^{(13,14)}$.

\section{ASPECTOS ÉTICOS}

Todos los estudios contaron con aprobación de un Comité de Ética (de la Universidad Peruana Cayetano Heredia o del Instituto de Investigación Nutricional).

\section{ANÁLISIS DE DATOS}

Se determinó la prevalencia de cada patotipo para cada estudio. Para la determinación de la prevalencia global de cada patotipo se consideró el promedio relativo de cada estudio con el 95\% de intervalo de confianza (IC $95 \%$ ). Para la comparación de las prevalencia por edad se utilizó la prueba de chi cuadrado.

\section{RESULTADOS}

Prevalencia de las E. coli diarrogénicas. Se analizaron 4243 muestras de diarrea (Tabla 2) y 3760 muestras de control (Tabla 3). La prevalencia de cada patógeno varió según la edad de la población de estudio y el tipo de estudio. En general, en el caso de diarrea, se encontró que EPEC y EAEC fueron los patógenos más frecuentes en cuatro estudios cada uno, seguido de ETEC, que fue el patógeno más frecuente en solo un estudio.

La prevalencia promedio global de los principales patógenos en muestras de diarrea fue EAEC 9,9\%, EPEC $8,5 \%$, ETEC 6,9\% y DAEC 4,8\% (Tabla 2 ). Cabe señalar algunas particularidades de cada estudio: en niños con

Tabla 2. E. coli diarrogénicas aisladas de muestras de diarrea en niños peruanos.

\begin{tabular}{|c|c|c|c|c|c|c|c|c|}
\hline N. ${ }^{\circ}$ & Estudio (Investigadores, año) & $\begin{array}{l}\mathrm{N} \cdot{ }^{\circ} \text { de muestras } \\
\text { de diarrea }\end{array}$ & $\begin{array}{c}\text { EAEC } \\
\%\end{array}$ & $\begin{array}{c}\text { EPEC } \\
\%\end{array}$ & $\begin{array}{c}\text { ETEC } \\
\%\end{array}$ & $\begin{array}{c}\text { DAEC } \\
\%\end{array}$ & $\begin{array}{c}\text { STEC } \\
\%\end{array}$ & $\begin{array}{c}\text { EIEC } \\
\%\end{array}$ \\
\hline 1 & Lanata, 1986-7 & 733 & 7,9 & 3,8 & 7,1 & 5,2 & 0,5 & 0,3 \\
\hline 2 & Zavaleta, 2004 & 556 & 13,5 & 11,0 & 7,7 & 8,6 & 0,9 & 0,4 \\
\hline 3 & Zavaleta, 2005 & 120 & 5,0 & 6,6 & 20,8 & 15,0 & 0 & 0 \\
\hline 4 & Ochoa y Lanata, 2006-7 & 936 & 15,1 & 7,6 & 3,2 & 4,6 & 0,5 & 0 \\
\hline 5 & Ochoa, Lanata y Huicho, 2008 & 193 & 8,3 & 15,6 & 14,6 & 2,6 & 0,5 & 0 \\
\hline 6 & Llanos, Lee y Lopez, 2007-8 & 135 & 5,2 & 10,4 & 7,4 & 7,4 & 8,9 & 9,6 \\
\hline 7 & Medina, Rivera y Romero, 2007-8 & 70 & 5,7 & 5,7 & 4,2 & 1,4 & 1,4 & 0 \\
\hline \multirow[t]{2}{*}{8} & Ochoa, Chea y Cleary, 2008-10 & 1500 & 7,4 & 9,5 & 6,7 & 2,7 & 0,3 & 0,6 \\
\hline & $\begin{array}{l}\text { Prevalencia global } \\
\text { (IC 95\%) }\end{array}$ & 4243 & $\begin{array}{c}9,9 \\
(9,0-10,7)\end{array}$ & $\begin{array}{c}8,5 \\
(7,6-9,3)\end{array}$ & $\begin{array}{c}6,9 \\
(6,1-7,6)\end{array}$ & $\begin{array}{c}4,8 \\
(4,2-5,5)\end{array}$ & $\begin{array}{c}0,8 \\
(0,5-1,0)\end{array}$ & $\begin{array}{c}0,6 \\
(0,4-0,8)\end{array}$ \\
\hline
\end{tabular}


Tabla 3. E. coli diarrogénicas aisladas de muestras control en niños peruanos sin diarrea.

\begin{tabular}{|c|c|c|c|c|c|c|c|c|}
\hline N. ${ }^{\circ}$ & Estudio (Investigadores, año) & $\begin{array}{c}\mathrm{N} .^{\circ} \text { de } \\
\text { muestras } \\
\text { control }\end{array}$ & $\begin{array}{l}\text { EAEC } \\
\%\end{array}$ & $\begin{array}{l}\text { EPEC } \\
\%\end{array}$ & $\begin{array}{c}\text { ETEC } \\
\%\end{array}$ & $\begin{array}{l}\text { DAEC } \\
\%\end{array}$ & $\begin{array}{l}\text { STEC } \\
\%\end{array}$ & $\begin{array}{c}\text { EIEC } \\
\%\end{array}$ \\
\hline 1 & Lanata, 1986-7 & 467 & 8,6 & 4,7 & 8,6 & 3,9 & 0,1 & 0,2 \\
\hline 4 & Ochoa y Lanata, 2006-7 & 424 & 17,9 & 9,9 & 1,2 & 2,1 & 1,2 & 0 \\
\hline 5 & Ochoa, Lanata y Huicho, 2008 & 318 & 12,9 & 17,0 & 8,8 & 1,9 & 0 & 0 \\
\hline 7 & Medina, Rivera y Romero, 2007-8 & 70 & 10,0 & 10,0 & 2,8 & 2,8 & 0 & 0 \\
\hline \multirow[t]{2}{*}{8} & Ochoa, Chea y Cleary, 2008-10 & 2,481 & 9,2 & 11,5 & 4,1 & 2,0 & 0,4 & 0,8 \\
\hline & $\begin{array}{l}\text { Prevalencia global } \\
\text { (IC 95\%) }\end{array}$ & 3,760 & $\begin{array}{c}10,4 \\
(9,4-11,4) \\
\end{array}$ & $\begin{array}{c}10,9 \\
(9,9-11,4) \\
\end{array}$ & $\begin{array}{c}4,7 \\
(4,0-5,4) \\
\end{array}$ & $\begin{array}{c}2,3 \\
(1,8-2,7) \\
\end{array}$ & $\begin{array}{c}0,5 \\
(0,3-0,8) \\
\end{array}$ & $\begin{array}{c}0,6 \\
(0,3-0,8) \\
\end{array}$ \\
\hline
\end{tabular}

diarrea y deshidratación el patógeno más frecuente fue ETEC, responsable del $20,8 \%$ de todos los episodios, seguido de DAEC (15,0\%) (estudio \# 3, Tabla 2). Por otro lado, en estudios comunitarios, con episodios de diarrea más leves, los patógenos más frecuentes fueron EPEC, EAEC y ETEC, aunque la frecuencia relativa de cada patógeno varió de estudio a estudio (estudios \# 1, 2, 4, 5 y 8 , Tabla 2). En niños con diarrea con sangre (estudio \# 6 , Tabla 2 ) la frecuencia relativa de STEC $(8,9 \%)$ y EIEC $(9,6 \%)$ fue mucho mayor en comparación a los estudios comunitarios que incluyen casos más leves de diarrea acuosa o disentérica. En niños infectados con el VIH, los patógenos más frecuentes fueron EAEC y EPEC (estudio \#7, Tabla 2). La frecuencia de estos patógenos varía según la edad del niño.

En la Figura 2, se presenta la distribución de las DEC según grupo de edad en pacientes con diarrea, tomando como referencia los estudio \# 4 y 5 , los cuales fueron estudios consecutivos llevados a cabo en el mismo lugar, con la misma metodología (vigilancia pasiva de diarrea), en 1034 niños seguidos de los 2 a los 12 meses de edad y en 529 niños de la misma primera cohorte

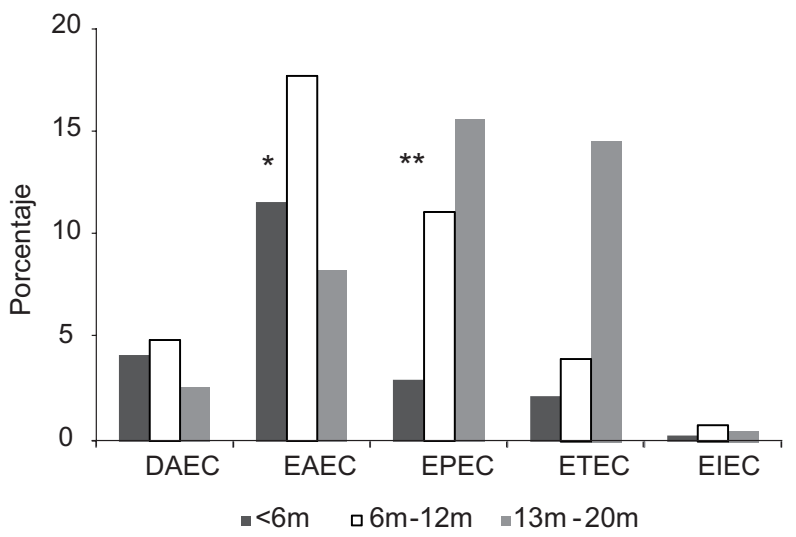

Figura 2. Frecuencia de las E. coli diarrogénicas en niños con diarrea según grupo de edad. Tamaño de la muestra: $<6 \mathrm{~m}$ $(n=406), 6 m-12 m(n=530), 13 m-20 m(n=193)$

${ }^{*} p<0,01 ;{ }^{* *} p<0,001$ seguidos subsecuentemente desde los 12 hasta los 20 meses de edad. En el caso de EPEC se encuentra que la prevalencia de estos patógenos aumenta significativamente con la edad $(p<0,001)$. En el caso de las muestras control tomadas de niños sin diarrea u otros síntomas gastrointestinales, los patógenos más frecuentes fueron: EPEC 10,9\%, EAEC 10,1\% y ETEC $4,7 \%$ (prevalencia promedio global) (Tabla 3).

Características clínicas de las infección por las $E$. coli diarrogénicas. En las muestras positivas a DEC, la coinfección con otros patógenos (otras entero-bacterias, virus $u$ otras DEC) fue más común en las muestras de diarrea que las de control (40\% frente a $16 \%)$ (10). Para la descripción de las características clínicas se consideraron los episodios en los que se identificó un solo patógeno (excluyendo coinfecciones). Se utilizaron los datos del estudio de cohorte (\#4), dado que este estudio tuvo un adecuado seguimiento clínico de cada episodio de diarrea. En general, se observó que los patotipos asociados con mayor duración de diarrea fueron DAEC y EPEC.

El mayor número de deposiciones líquidas por día se observó en EPEC y ETEC, los cuales presentaron un mayor puntaje de gravedad (Vesikari). La mitad de los niños con ETEC recibieron sales de rehidratación oral, similar a lo encontrado por rotavirus, patógeno con el mayor puntaje de severidad en esta misma población (10). Por otro lado, se determinó el número de leucocitos fecales por campo de elevado poder (L/c) en muestras positivas a DEC como infección única, excluyendo coinfecciones. Se encontró leucocitos fecales $>10$ L/c en 8,5\% (18/212) de muestras de diarrea frente a 1,3\% (2/157) de muestras control $(p<0,01)$; sin embargo, la respuesta inflamatoria fue leve en general para estos patógenos ${ }^{(15)}$.

Factores de virulencia y variabilidad genética de las $E$. coli diarrogénicas. Si bien las DEC son diagnosticadas por la presencia de genes específicos para cada patotipo, este grupo de bacterias son bastante heterogéneas dentro del mismo grupo. Se estudió la 
variabilidad genética en cepas aisladas de los estudios en la comunidad (estudios \# 4 y 5 ).

EPEC. En 120 cepas de EPEC se determinó la presencia y variabilidad alélica de tres genes de virulencia: eae (intimina, necesaria para la unión íntima de la bacteria al enterocito), bfpA (subunidad del pilus necesario para la adherencia localizada) y perA (regulador de la transcripción) mediante PCR-RFLP (restriction fragment length polymorphism) ${ }^{(16)}$. Se encontró que las EPECatípicas (eae+, bfp-) fueron el tipo más frecuente, tanto en muestras de diarrea $(54 / 74,73 \%)$ como en muestras control (40/46, 87\%). Se encontraron 13 alelos de intimina (eae), los más frecuentes fueron: beta, theta y kappa; cinco alelos de $b f p A$, los más frecuentes fueron beta1/7 y alpha3; y tres alelos de perA, los más frecuentes fueron beta y alpha. El alelo gamma de intimina se encontró con más frecuencia en los episodios de mayor duración que en los cortos; el alelo kappa de intimina estuvo asociado a episodios con mayor gravedad. La cepas pertenecieron a 36 serogrupos distintos; los más frecuentes fueron: 055 (7\%), $91(3 \%), 128(3 \%)$ y $153(3 \%)$. Un total de 51 cepas fueron O-no tipificables (44\%) ${ }^{(16)}$.

ETEC. Se estudiaron las características genotípicas y fenotípicas en 85 cepas. El tipo más frecuente fue ETEC-LT (56\%), seguido de ETEC-ST (25\%) y ETECLT/ST (19\%). Se estudiaron los factores de colonización (CFA o CF, fimbrias necesarias para colonización) por DOT-BLOT ELISA usando 21 anticuerpos monoclonales. Los principales factores de colonización encontrados fueron CS6 (12\%), CS12 (9\%), CS1 (7\%), CS7 (5\%) y CS17 (5\%). Sin embargo, no se detectaron factores de colonización en $43 \%$ de las cepas de diarrea y $67 \%$ de las muestras control ${ }^{(17)}$.

EAEC. Se evaluó la presencia de 22 genes de virulencia en 178 cepas (102 aisladas de casos de diarrea y 76 de muestras control). Los genes con mayor frecuencia fueron: fyuA (yersiniabactina) presente en $88 \%$, seguido de los genes asociados a la dispersión: aatA (proteína transportadora de dispersina) en $84 \%$ y aap (dispersina) en $73 \%$. Entre las toxinas hubo una mayor frecuencia de set1A (enterotoxina de shigella 1A) presente en $55 \%$ (Durand D, comunicación personal).

DAEC. En 25 cepas DAEC aisladas de niños con diarrea se estudiaron los patrones de adherencia y polimerización de actina en cultivo de células HEp-2. El patrón de adherencia difusa estuvo presente en el $88 \%$ de las cepas, mientras que la acumulación o polimerización de actina estuvo presente en el $60 \%$. Estos resultados deben confirmarse con un mayor número de cepas, así como la determinación de los genes de virulencia en estas cepas ${ }^{(18)}$.
STEC. Se estudió 29 cepas STEC (14 aisladas de casos de diarrea y 15 de muestras de control). Ninguno de los pacientes desarrolló síndrome urémico hemolítico u otra complicación de la infección por STEC. Se encontró toxina shiga 1 (sxt1) en el $83 \%$ de las cepas, sxt2 en $17 \%$ y eae en $72 \%$. Los serotipos más frecuente fueron $\mathrm{O} 26: \mathrm{H} 11$ (14\%), O111 (H10, H8 y H7) (10\%) y O103:H2 (7\%). No se encontró cepas de STEC con el serotipo virulento 0157:H7. Se determinó la relación filogenética de estas cepas usando MLST (Multilocus sequence typing) y PFGE (Pulsed-field gel electrophoresis). Se encontró una gran diversidad genética (13 tipos de secuencia por MLST y 19 patrones de campo pulsado) (19).

Resistencia antibiótica de las $E$. coli diarrogénicas. En las cepas estudiadas se encontró que las DEC en conjunto presentan elevados porcentajes de resistencia a antibióticos comúnmente usados como ampicilina (85\%), cotrimoxazol (79\%), tetraciclina (65\%) y ácido nalidíxico (28\%) ${ }^{(20)}$. En general la resistencia fue mayor en las cepas aisladas de pacientes con diarrea que en las cepas aisladas de pacientes asintomáticos. Así por ejemplo, la resistencia a ampicilina fue $85 \%$ en diarrea frente a $70 \%$ en cepas de control; la resistencia a cotrimoxazol fue $79 \%$ frente a $61 \%$, respectivamente; lo que refleja una mayor exposición a antibióticos en pacientes con gastroenteritis. Por otro lado, se encontró mayor resistencia en las cepas DAEC y EAEC, que en las cepas de EPEC y ETEC ${ }^{(20)}$.

Se ha estudiado los principales mecanismos moleculares de resistencia hacia cuatro familias de antibióticos: quinolonas, betalactámicos, cloranfenicol y tetraciclinas. En cuanto a la resistencia a quinolonas se estudió un total 52 cepas DEC con resistencia alta e intermedia hacia ácido nalidíxico. Se encontró mutaciones a nivel de gyrA en el $64 \%$ de cepas, mientras que para mutaciones a nivel de parC se encontró 5,8\% (13). La resistencia hacia las otras tres familias de antibióticos se estudió en un total de 85 cepas DEC ampicilina-resistentes de muestras de diarrea. Los principales genes de resistencia encontrados fueron: blaTEM (resistencia a betalactámicos) presente en 35\%, cat (resistencia a cloranfenicol) presente en $87 \%$, y tetA (resistencia a tetraciclina) en $31 \%$ y tetB en $21 \%{ }^{(15)}$.

Diagnóstico de las $E$. coli diarrogénicas. $E l$ diagnóstico de las DEC por métodos moleculares no se realiza como trabajo diario en los laboratorios clínicos. En casos de diarrea infantil, en muchos laboratorios se hace el diagnóstico presuntivo de EPEC en base a la determinación de los serotipos por aglutinación con antisueros $\mathrm{O}$ y $\mathrm{H}$. Sin embargo, este no es un método preciso, debido a que puede haber falsos positivos $y$ falsos negativos. Recientemente se realizó un estudio en 
113 cepas aisladas de niños e identificadas como "EPEC" en cuatro laboratorios clínicos de Lima. En estas cepas se determinó la presencia del gen de intimina (eae, usado en el diagnóstico molecular de EPEC), y se encontró solo 15 cepas $(13,3 \%)$ con el diagnóstico correcto de EPEC. Además se identificaron tres cepas ETEC, tres cepas STEC, una cepa EAEC y una cepa EIEC ${ }^{(21)}$.

\section{DISCUSIÓN}

En este estudio las E. coli diarrogénicas se encontraron en más de un tercio de las muestras estudiadas, representando, en conjunto, el principal grupo de patógenos en niños con diarrea en nuestro medio. La tasa de aislamiento y frecuencia relativa de cada patógeno varía según la edad del paciente y según el tipo de estudio (comunitario frente a hospitalario; comunitario de vigilancia pasiva frente a vigilancia activa de diarrea). La prevalencia relativa varía en relación a la gravedad de los episodios en cada uno de estos estudios. Así por ejemplo, se considera que la prevalencia relativa de patógenos en infecciones comunitarias es un marcador de la morbilidad de diarrea, mientras que la prevalencia de patógenos en pacientes hospitalizados se relaciona a los casos más graves, asociados con mortalidad por diarrea ${ }^{(5)}$. En este estudio encontramos que EPEC y EAEC fueron los patógenos más frecuentes en la comunidad, mientras que ETEC fue el más frecuente en pacientes hospitalizados con diarrea y deshidratación, lo cual se explica por su mecanismo de patogenia, al tener las toxinas termoestable (ST) y termolábil (LT). Está ultima tiene una gran homología a la toxina colérica que produce diarrea profusa y deshidratación. Si bien la frecuencia de cada patógeno varía de estudio en estudio, en general, los principales patotipos de E. coli encontrados en este estudio fueron EPEC, EAEC y ETEC, lo que concuerda con los estudio regionales y globales de las DEC en niños ${ }^{(22-27)}$.

En este estudio también encontramos una alta prevalencia de las DEC en muestras control tomadas de niños asintomáticos. La interpretación de la frecuencia de patógenos en muestras de diarrea frente a las de control, es bastante compleja. La colonización asintomática puede ser el resultado de varios factores (del patógeno, del huésped o del ambiente). Uno podría argumentar de manera simplista, que si un patógeno se encuentra con la misma frecuencia en muestras control que miden colonización, entonces muy probablemente este patógeno no sea una causa verdadera de diarrea. Sin embargo, la presencia de un patógeno en muestras de heces en niños asintomáticos puede ser el resultado de factores del huésped, como la edad, lo cual puede estar relacionado con factores protectores en edad temprana, como la lactancia, los anticuerpos transplacentarios o factores protectores a mayor edad, como el desarrollo de inmunidad natural en función a las infecciones previas (lo cual es la base para el desarrollo, por ejemplo de la vacuna para ETEC, como lo fue para la de rotavirus).

Por otro lado, están los factores del patógeno: no todas las cepas son iguales. Por ejemplo, en el caso de EPEC y EAEC, hemos descrito la gran heterogeneidad en la presencia de genes de virulencia. Es decir, dos cepas pueden ser categorizadas como EAEC, pero su carga genética y de virulencia puede ser completamente diferente. Actualmente nuestro grupo de investigación está centrado en el estudio de genes o grupo de genes asociados con cuadros de diarrea más graves o de mayor duración. Esto, a la larga, podría llevar a la identificación de marcadores de severidad para un diagnóstico más certero de las cepas clínicas verdaderamente virulentas. Adicionalmente, la presencia de coinfecciones hace complicado determinar cuál es el patógeno responsable de la enfermedad o si hay un efecto aditivo de cada patógeno presente en la infección mixta. Esto representa un problema pobremente definido en la epidemiología actual de diarrea. Finalmente, están los factores del ambiente. No es lo mismo medir la colonización con patógenos entéricos en niños de zonas periurbanas, con gran exposición a contaminación de agua y alimentos, que niños de de ciudades desarrolladas.

Otro aspecto importante de este estudio, son las altas tasas de resistencia antibiótica encontrada, especialmente a ampicilina y cotrimoxazol. Estos dos antibióticos no deben usarse para el manejo empírico inicial de diarrea bacterianas en nuestro medio (estos antibióticos también presentan alta frecuencia de resistencia en cepas de Shigella localmente). El problema de resistencia a antimicrobianos es particularmente críticos en países en vías de desarrollo. Otros estudios con cepas de DEC han reportado frecuencias elevadas de resistencia en Vietnam ${ }^{(28)}$, Tanzania ${ }^{(29)}$, México ${ }^{(30)}$, similar al estudio nuestro ${ }^{(20)}$.La determinación de los mecanismos moleculares de resistencia, muestra la gran diversidad de mecanismos y genes asociados, permitiendo el desarrollo de estudios de mecanismos de transmisión y diseminación de la resistencia antimicrobiana.

El gran reto en el estudio de las DEC, es poder contar con métodos de diagnósticos más fáciles, rápidos y baratos, que se puedan usar tanto en los laboratorios clínicos como en estudios comunitarios. Como se presentó en los resultados, actualmente no se debería usar la serología para la determinación de las DEC, sobre todo para EPEC, dado a su baja sensibilidad y especificidad (21). La serología sí es ideal para estudio de brotes por ejemplo, pues se puede identificar cepas específicas. 
Mientras no se desarrollen estas pruebas rápidas (tipo inmunocromatografía), el estudio de las DEC seguirá centrado en laboratorios de investigación.

Una de las limitaciones de este estudio es que la prevalencia real de estos patógenos pueda que sea más alta, dado que en tres estudios trabajamos con cepas guardadas. Por lo tanto, algunos genes, sobre todo los plasmídicos, pueden haberse perdido en el almacenamiento. Otra limitación es la ausencia de datos clínicos en todos los estudios, lo que hubiera permitido comparar prevalencia de cada patotipo según las características clínicas de la infección. Sin embargo, este trabajo representa uno de los estudios más grandes de diarrea en niños (más de 4000 muestras de diarrea), en los que de manera sistemática se buscaron todas las E. coli diarrogénicas con la misma metodología en todas y cada una de las cepas de $E$. coli.

En conclusión, las DEC son causa importante de diarrea en niños peruanos, sin embargo, su diagnóstico no se realiza como trabajo diario en los laboratorios clínicos. Estos patógenos son altamente heterogéneos y es necesario complementar estudios de virulencia y variabilidad alélica, para comprender mejor el rol de cada patógeno como causa de diarrea. A nivel nacional se requieren estudios adicionales para determinar la prevalencia en otras regiones del Perú, sobre todo en zonas rurales de la sierra y la selva, así como estudios en casos graves de diarrea en niños hospitalizados, y trabajos que permitan estudiar la transmisión de estos patógenos en muestras de animales, agua y alimentos.

\section{AGRADECIMIENTOS}

A los doctores: Claudio F. Lanata (Instituto de Investigación Nutricional, Lima, Perú; Universidad Peruana de Ciencias Aplicadas, Lima, Perú) por habernos proporcionado las cepas de Huaraz (estudio \#1) y el financiamiento y conducción de los estudios del cono sur de Lima (estudios \# 4 y 5); Nelly Zavaleta (Instituto de Investigación Nutricional, Lima, Perú) por las cepas de Villa el Salvador y del Instituto Nacional de Salud del Niño (estudios \# 2 y 3); Luis Huicho (Universidad Peruana Cayetano Heredia, Universidad Nacional Mayor de San Marcos, Instituto Nacional de Salud del Niño, Lima, Perú) por el financiamiento de la segunda cohorte del estudio del cono sur de Lima (estudio \# 5); Alejandro Llanos, Jorge Lee y Francisco López (Universidad Peruana Cayetano Heredia) por las cepas del estudio del Hospital Nacional Cayetano Heredia (estudio \# 6); Anicia Medina y Liliana Romero (Universidad Peruana Cayetano Heredia) por las cepas en pacientes VIH (estudio \# 7); Elsa Chea (Universidad Peruana Cayetano Heredia,) y Thomas G. Cleary (Uni- versity of Texas School of Public Health, Houston, USA) por las cepas del estudio de Independencia (estudio \# 8); y Eric R. Hall y Ryan Maves (Naval Medical Research Center Detachment, Lima, Perú) por la colaboración en los estudios con ETEC y conservación de las cepas.

\section{Fuentes de Financiamiento}

Este trabajo fue financiado por el Fondo Concursable de la Universidad Peruana Cayetano Heredia (T. Ochoa) y del Instituto Nacional de Salud del Niño (T. Ochoa y $L$. Huicho); Grant 1K01TW007405 del Instituto Nacional de Salud de los Estados Unidos (T. Ochoa); y por la Agencia Española de Cooperación Internacional para el Desarrollo (AECID) (T. Ochoa y J. Ruiz). J.Ruiz dispone de un contrato Miguel Servet (CP05/0130) del Fondo de Investigaciones Sanitarias.

\section{Conflictos de Interés}

Los autores declaran no tener conflictos de interés en la publicación del presente artículo.

\section{REFERENCIAS BIBLIOGRÁFICAS}

1. Boschi-Pinto C, Velebit L, Shibuya K. Estimating child mortality due to diarrhoea in developing countries. Bull World Health Organ. 2008;86(9):710-7.

2. Nataro JP, Kaper JB. Diarrhoegenic Escherichia coli. Clin Microbiol Rev. 1998;11(1):142-201.

3. Guion CE, Ochoa TJ, Walker CM, Barletta F, Cleary TG. Detection of diarrheagenic Escherichia coli by use of melting-curve analysis and real-time multiplex PCR. J Clin Microbiol. 2008;46(5):1752-7.

4. O’Ryan M, Prado V, Pickering LK. A millennium update on pediatric diarrheal illness in the developing world. Semin Pediatr Infect Dis. 2005;16(2):125-36.

5. Lanata CF, Mendoza W, Black RE. Improving diarrhoea estimates. Geneva: WHO; 2002. Disponible en: http://www. who.int/child_adolescent_health/documents/pdfs/improving_diarrhoea_estimates.pdf. Fecha de acceso: 21 de abril de 2009.

6. Cama RI, Parashar UD, Taylor DN, Hickey T, Figueroa D, Ortega YR, et al. Enteropathogens and other factors associated with severe disease in children with acute watery diarrhea in Lima, Peru. J Infect Dis. 1999;179(5):1139-44.

7. Arias I, Huguet JC. Detección Molecular de Toxinas Termoestable y Termolabil de Escherichia coli mediante Hibridación. Rev Peru Med Exp Salud Publica. 2002;19(4):193-6.

8. Arias I, Cáceres O, Figueroa M. Huguet J, Camiña M. Escherichia coli enteroagregativa en niños con diarrea de un hospital de Lima. Rev Peru Med Exp Salud Publica. 2004;21(3):176-8.

9. Zavaleta N, Figueroa D, Rivera J, Sánchez J, Alfaro S, Lönnerdal B. Efficacy of rice-based oral rehydration solution containing recombinant human lactoferrin and lysozyme in Peruvian children with acute diarrhea. J Pediatr Gastroenterol Nutr. 2007;44(2):258-64. 
10. Ochoa TJ, Ecker L, Barletta F, Mispireta ML, Gil Al, Contreras C, et al. Age related susceptibility to infection with diarrheagenic Escherichia coli among infants from periurban areas in Lima, Peru. Clin Infect Dis. 2009;49(11):1694-702.

11. Medina AM, Rivera FP, Romero LM, Kolevic LA, Castillo ME, Verne E, et al. Diarrheagenic Escherichia coli in HIV pediatric patients in Lima, Perú. Am J Trop Med Hyg. 2010;83(1):158-63.

12. Barletta F, Ochoa TJ, Ecker L, Gil Al, Lanata CF, Cleary TG. Validation of five-colony pool analysis using multiplex real-time PCR for detection of diarrheagenic Escherichia coli. J Clin Microbiol. 2009;47(6):1915-7.

13. Pons MJ, Mosquito S, Mercado E, Rivera FP, Maves R, Del Valle LJ, et al. Quinolone resistance mechanisms in Escherichia coli isolated from children (with or without diarrhoea) under 1 year in Lima, Perú. Presentation at 20th European Congress of Clinical Microbiology and Infectious Diseases. Viena - Austria. 2010; 10-13 April. [abstract no. 2809]

14. Mosquito SG. Mecanismos de Resistencia Antibiótica en cepas de Escherichia coli comensales y diarrogénicas aisladas de niños peruanos menores de 2 años. [Tesis de Maestría]. Lima: Facultad de Ciencias y FilosofíaUniversidad Peruana Cayetano Heredia; 2011.

15. Mercado EH, Ochoa TJ, Ecker L, Cabello M, Durand D, Barletta F, et al. Fecal leukocytes in children infected with diarrheagenic E. coli. J Clin Microbiol. 2011 Feb 16. [Epub ahead of print] doi: 10.1128/JCM.02199-10.

16. Contreras CA, Ochoa TJ, Lacher DW, DebRoy C, Navarro A, Talledo M, et al. Allelic variability of critical virulence genes (eae, bfpA, and perA) in typical and atypical EPEC in Peruvian children. J Med Microbiol. 2010;59:25-31.

17. Rivera FP, Ochoa TJ, Maves RC, Bernal M, Medina AM, Meza R, et al. Genotypic and phenotypic characterization of enterotoxigenic Escherichia coli (ETEC) strains isolated from Peruvian children. J Clin Microbiol. 2010;48(9):3198-203.

18. Riveros M, Barletta F, Cabello M, Durand D, Mercado EH, Contreras CA, et al. Patrones de adherencia de cepas de Escherichia coli difusamente adherente (DAEC) provenientes de niños con y sin diarrea. Rev Peru Med Exp Salud Publica. 2011; 28(1). [En prensa]

19. Contreras CA, Ochoa TJ, Ruiz J, Lacher DW, Rivera FP, Saenz Y, et al. Phylogenetic relationships of Shiga toxinproducing Escherichia coli (STEC) isolated from Peruvian children. J Med Microbiol. 2011 Feb 3. [Epub ahead of print] doi: 10.1099/JMM.0.026666-0.

20. Ochoa TJ, Ruiz J, Molina M, Del Valle LJ, Vargas M, Gil Al, et al. High frequency of antimicrobial drug resistance of diarrheagenic Escherichia coli in infants in Peru. Am J Trop Med Hyg. 2009;81(2):296-301.

21. Lluque A, Mercado E, Riveros M, Alvarado L, Carlos E, Colichón A, et al. Comparison of enteropathogenic Escherichia coli (EPEC) diagnosis by serology and by polymerase chain reaction (PCR). Rev Gastroenterol Perú. 2010;30(2):121-5.
22. Levine MM, Ferreccio C, Prado V, Cayazzo M, Abrego $\mathrm{P}$, Martinez J, et al. Epidemiologic studies of Escherichia coli diarrheal infections in a low socioeconomic level periurban community in Santiago, Chile. Am J Epidemiol. 1993; 138(10):849-69.

23. Porat N, Levy A, Fraser D, Deckelbaum RJ, Dagan R. Prevalence of intestinal infections caused by diarrheagenic Escherichia coli in Bedouin infants and young children in Southern Israel. Pediatr Infect Dis J. 1998;17(6):482-8.

24. Quiroga M, Oviedo P, Chinen I, Pegels E, Husulak E, Binztein N, et al. Asymptomatic infections by diarrheagenic Escherichia coli in children from Misiones, Argentina, during the first twenty months of their lives. Rev Inst Med Trop Sao Paulo. 2000;42(1):9-15.

25. Sarantuya J, Nishi J, Wakimoto N, Erdene S, Nataro JP, Sheikh J, et al. Typical enteroaggregative Escherichia coli is the most prevalent pathotype among $\mathrm{E}$. coli strains causing diarrhea in Mongolian children. J Clin Microbiol. 2004;42(1):133-9.

26. Araujo JM, Tabarelli GF, Aranda KR, Fabbricotti SH, Fagundes-Neto $\mathbf{U}$, et al. Typical enteroaggregative and atypical enteropathogenic types of Escherichia coli are the most prevalent diarrhea-associated pathotypes among Brazilian children. J Clin Microbiol. 2007;45(10):3396-9.

27. Spano LC, Sadovsky AD, Segui PN, Saick KW, Kitagawa SM, Pereira FE, et al. Age-specific prevalence of diffusely adherent Escherichia coli in Brazilian children with acute diarrhoea. J Med Microbiol. 2008;57:359-63.

28. Nguyen TV, Le Van P, Le Huy C, Gia KN, Weintraub A. Detection and characterization of diarrheagenic Escherichia coli from young children in Hanoi, Vietnam. J Clin Microbiol. 2005;43(2):755-60.

29. Vila J, Vargas M, Casals C, Urassa H, Mshinda $H$, Schellemberg D, et al. Antimicrobial resistance of diarrheagenic Escherichia coli isolated from children under the age of 5 years from Ifakara, Tanzania. Antimicrob Agents Chemother.1999; 43(12):3022-4.

30. Estrada-García T, Cerna JF, Pacheco-Gil L, Velázquez RF, Ochoa TJ, Torres $\mathbf{J}$, et al. Drug-resistant diarrheogenic Escherichia coli, Mexico. Emerg Infect Dis. 2005;11(8):1306-8.

Correspondencia: Theresa J. Ochoa

Dirección: Instituto de Medicina Tropical Alexander von Humboldt. Universidad Peruana Cayetano Heredia.

Av. Honorio Delgado 430, San Martín de Porras, Lima 33, Perú Teléfono: (511) 482-3903 / (511) 482-3404.

Correo electrónico: Theresa.J.Ochoa@uth.tmc.edu; Theresa. Ochoa@upch.pe 